\title{
In-stent restenosis of drug-eluting stents: clinical presentation and outcomes in a real-world scenario
}

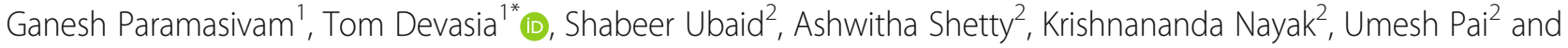
Mugula Sudhakar Rao ${ }^{1}$

\begin{abstract}
Background: Drug-eluting stents (DES) have substantially reduced the incidence of coronary in-stent restenosis (ISR), but the problem persists. Clinical presentation and outcomes of DES-ISR in a real-world scenario remains underreported.
\end{abstract}

Results: In this retrospective study, we examined medical records of 191 consecutive patients with DES-ISR (210 ISR lesions) hospitalized between January 2013 and December 2017. ISR clinical presentation was classified as acute coronary syndrome (ACS) or non-ACS. Clinical, angiographic features and 1-year outcomes [composite of death, myocardial infarction (MI) and repeat-target lesion revascularization] for these two groups were compared. The mean age of study population was $61 \pm 10$ years and $81.2 \%$ were males. ACS was the dominant clinical presentation mode occurring in 118 (61.8\%) patients. Ml was seen in 66 (34.6\%) patients. Female gender (odds ratio, 2.71 ; $95 \%$ confidence interval $[\mathrm{Cl}], 1.13-6.52 ; P=0.026$ ) and chronic kidney disease (odds ratio, 3.85; 95\% Cl, $1.05-$ $14.20 ; P=0.043)$ correlated significantly with ACS ISR presentation. A majority [104 (54.5\%)] of patients underwent percutaneous coronary intervention $(\mathrm{PCl})$, of whom $72(69.2 \%)$ received a new DES. The rest either underwent CABG (26.2\%) or received medical therapy (19.4\%). Patients presenting with ACS had a significantly worse clinical outcome at 1-year follow-up (ACS versus non-ACS presentation: hazard ratio [HR], 2.66; 95\% Cl, 1.09-6.50; $P=0.032$ ).

Conclusions: DES-ISR presents most commonly as ACS. Female gender and chronic kidney disease seem to be associated with ACS presentation. ACS presentation of ISR is associated with worse 1-year outcomes. Early identification of those with ACS risk and closer follow-up may improve outcomes.

Keywords: In-stent restenosis, Drug-eluting stent, Percutaneous coronary intervention

\section{Background}

Restenosis is the Achilles' heel of coronary intervention [1]. Bare-metal stents (BMS) reduced the restenosis rates seen with balloon angioplasty predominantly by mitigating the effects of elastic recoil and negative remodelling $[2,3]$. However, the vascular response to injury in the form of neointimal hyperplasia offset the aforementioned benefits and led to in-stent restenosis (ISR) [4]. Introduction of DES, which was designed to minimize this problem, resulted in two important changes. First,

\footnotetext{
*Correspondence: gt.cardiology@gmail.com

'Department of Cardiology, Kasturba Medical College, Manipal, Manipal

Academy of Higher Education, Manipal, Karnataka 576104, India

Full list of author information is available at the end of the article
}

the incidence of ISR reduced to $5-10 \%$ compared to BMS era where up to a third of the patients presented with restenosis [5-9]. Second, focal angiographic ISR pattern became more common compared to BMS ISR which presented commonly with a diffuse pattern [10]. Considering the increasing DES usage in the present era worldwide, even this lower ISR incidence with DES still accounts for a large number of cases yearly.

Initial studies of DES ISR focused on the occurrence of angiographic ISR, but a significant proportion of ISR cases remain asymptomatic and thus may not be relevant clinically. Clinical ISR (presence of symptoms or objective evidence of myocardial ischemia attributable to ISR) presents a therapeutic challenge and carries prognostic implications. 
Only a few studies have explored the relationship between the types of ISR clinical presentation and outcomes mostly following a retrospective design. Most such studies have included only those patients who underwent percutaneous coronary intervention (PCI) for culprit ISR lesion [11, 12]. Since in the real world scenario patients may be treated with medical therapy, percutaneous or surgical revascularization, these studies possess an inherent selection bias and may not be representative of the entire clinical ISR population. We set out to study clinical ISR of DES in the realworld scenario, focusing on the presentation mode and its impact on clinical outcomes.

\section{Methods}

\section{Study population and design}

This single-centre, retrospective cohort study included consecutive patients with clinical culprit ISR lesions of drug-eluting stents (DES) diagnosed between January 2013 and December 2017 at a tertiary care hospital in South India (Fig. 1). Approval for the study protocol was obtained from the institutional ethics committee.

Medical records of these patients were examined. Clinical and demographic characteristics including risk factors for atherosclerotic cardiovascular disease (ASCVD) and investigations including results of blood tests, electrocardiography and echocardiography tests were recorded. Angiographic images were reviewed by two independent cardiologists who confirmed the presence of ISR and also determined the ISR type (as described by Mehran et al.) [13]. Details of treatment given for the culprit ISR lesion, including interventional procedures, were noted. Also, details of initial PCI (prior to ISR diagnosis), when available, including the type of DES used were recorded.

\section{Study definitions}

In-stent restenosis was defined angiographically as the presence of $>50 \%$ diameter stenosis at the stent site or at its edges (adjacent $5 \mathrm{~mm}$ segments) [14]. Clinical ISR was diagnosed when symptoms and/or inducible ischemia (on stress tests) were present and were attributable to the ISR lesion.

Index hospitalization was the hospitalization event during which ISR was first diagnosed. Clinical presentation during this hospitalization was classified into ACS (acute coronary syndrome) which included patients with unstable angina (UA) and myocardial infarction (MI), and non-ACS which included patients diagnosed with either stable angina or silent ischemia.

Diagnosis of MI was based on the universal definition and was categorized into STEMI (ST-elevation myocardial infarction) and NSTEMI (non-ST elevation myocardial infarction) [15]. Typical chest discomfort brought upon by physical exertion and relieved by rest and/or nitrates was diagnosed as stable angina. Unstable angina

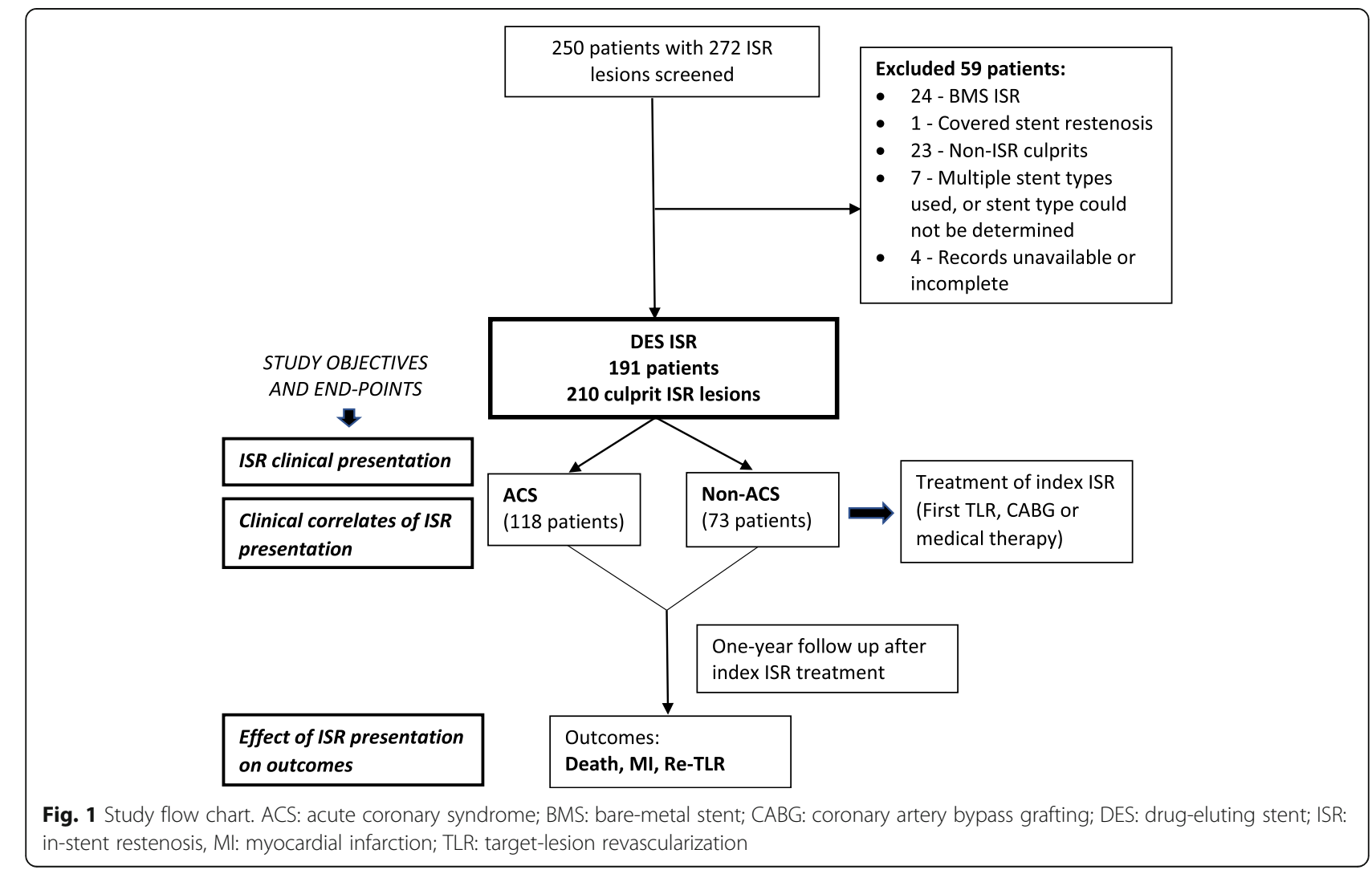


was defined as a recent onset or worsening of typical chest pain, chest pain lasting $>20 \mathrm{~min}$ and/or occurring at rest with or without ST-segment changes on electrocardiography and without an increase in the blood levels of cardiac biomarkers. Silent ischemia was defined as ischemia identified on stress tests (treadmill exercise test or dobutamine echocardiography) in the absence of symptoms [16].

The presence of chronic kidney disease was established using the definitions provided in the Kidney Disease: Improving Global Outcomes (KDIGO) 2012 guidelines [17]. Stent thrombosis was diagnosed using the criteria suggested by the Academic Research Consortium as definite or probable [18].

\section{Outcome definitions}

The primary outcome was a composite outcome which included major adverse cardiac events (MACE) and mortality from all causes following index hospitalization. MACE included both repeat MI and repeat TLR (target-lesion revascularization) which occurred during the 1-year followup period. MACE did not include the revascularization procedure carried out for index ISR lesion or the MI event which led to index hospitalization. All deaths were assumed to be cardiac unless another clearly documented cause was available.

\section{Follow-up}

Follow-up data for a duration of 1 year following the index hospitalization was obtained from patients' hospital records to determine the occurrence of one or more of the outcome events defined above. Telephonic contact was used to collect follow-up data for patients in whom such data was not available from hospital/medical records. Two independent cardiologists who were blinded to the study objectives adjudicated all the outcome events.

\section{Study objectives}

The main objective was to study the clinical presentation and its impact on the occurrence of MACE in patients with ISR in DES. The secondary objective was to determine the clinical correlates for ACS as the presentation mode for DES-ISR compared to non-ACS (Fig. 1).

\section{Statistical analysis}

Continuous variables were described using mean and standard deviation. Frequencies (\%) were used for categorical variables. Chi-square test or Fisher's exact test was used for categorical variables and $t$ test for continuous variables to compare groups with ACS and non-ACS presentations. Logistic regression analysis (univariate and multivariate) was used to test correlates for ACS as ISR presentation. Survival (time-to-event) analysis for adverse events according to ISR presentation mode was carried out using the Kaplan-Meier method and the log-rank test. Cox regression analysis was used to calculate the hazard ratio for the effect of clinical presentation mode on outcomes. $P$ value of $<0.05$ was taken as an indicator of statistical significance. Statistical analysis was carried out using SPSS software (version 16.0).

\section{Results}

\section{Clinical and angiographic characteristics}

This study included 191 patients with 210 culprit ISR lesions. Mean $( \pm \mathrm{SD})$ age of the patients was $61( \pm 10)$ years and $155(81.2 \%)$ were males. Patient characteristics, stratified according to clinical presentation at the time of index hospitalization, are represented in Table 1. ACS was the dominant clinical presentation mode, occurring in $118(61.8 \%)$ patients. Among patients presenting with ACS, 52 (44\%) had UA, 52 (44\%) had NSTEMI and $14(12 \%)$ had STEMI. Among the $73(38.2 \%)$ patients who presented as non-ACS, $63(86.3 \%)$ had stable angina and the remaining 10 (13.7\%) had silent ischemia which was diagnosed during stress testing. ACS cohort had a significantly higher proportion of women and patients with CCF and chronic kidney disease.

Table 2 shows the angiographic characteristics, treatment strategy and characteristics of interventional procedures. For patients who underwent PCI for culprit ISR lesions, the details of the type of intervention including the type of stents and adjunctive devices used are shown. Focal ISR lesions were more common than all other ISR lesion types combined (63.8\% vs. $36.2 \%)$. Type $1 \mathrm{C}$ Mehran type was the most common type of ISR lesion found in both ACS and non-ACS groups (34.1\% and 35.8\% respectively). There was no difference between the type of ISR lesions found in ACS and non-ACS groups $(P=$ 0.961). Both groups were similar with respect to disease burden, vessels affected by ISR, ISR location and treatment received. PCI was the most common treatment modality in both groups, and more than two thirds of those patients received a new DES.

\section{Clinical correlates for ACS as ISR presentation}

We analysed the clinical correlates for ACS as ISR presentation (Table 3). Female gender (odds ratio, 2.71; 95\% CI, $1.13-6.52 ; P=0.026)$ and chronic kidney disease (odds ratio, 3.85; $95 \% \mathrm{CI}, 1.05-14.20 ; P=0.043$ ) correlated with ACS presentation. Patients with ACS were more likely to have CCF (odds ratio, 4.98; 95\% CI, 1.63-15.26; $P=$ 0.005). Age, body mass index, diabetes mellitus, hypertension, dyslipidemia, tobacco use, previous MI or previous CABG did not correlate with ACS ISR presentation. Lesion-related characteristics like proximal ISR location or involvement of the left anterior descending artery also did not correlate with an ACS presentation. 
Table 1 Patient characteristics at first clinical ISR presentation

\begin{tabular}{|c|c|c|c|c|}
\hline Parameter & Total $(n=191)$ & ACS cohort $(n=118)$ & Non-ACS cohort $(n=73)$ & $P$ value \\
\hline \multicolumn{5}{|l|}{ Demographics } \\
\hline Age & $61 \pm 10$ & $62 \pm 10$ & $59 \pm 10$ & 0.074 \\
\hline Men & $155(81.2 \%)$ & $90(76.3 \%)$ & 65 (89.0\%) & 0.036 \\
\hline BMI & $23.5 \pm 3.5$ & $23.7 \pm 3.9$ & $23.1 \pm 2.7$ & 0.479 \\
\hline \multicolumn{5}{|l|}{ Clinical characteristics } \\
\hline Diabetes & $109(57.1 \%)$ & $68(57.6 \%)$ & $41(56.2 \%)$ & 0.881 \\
\hline Hypertension ${ }^{t}$ & $104(54.5 \%)$ & $66(55.9 \%)$ & $38(52.1 \%)$ & 0.655 \\
\hline Chronic kidney disease & $21(11.0 \%)$ & $18(15.3 \%)$ & $3(4.1 \%)$ & 0.017 \\
\hline Acute kidney injury & $32(16.8 \%)$ & $24(20.3 \%)$ & $8(11.0 \%)$ & 0.112 \\
\hline Dyslipidemia* & $98(67.1 \%)$ & $55(63.2 \%)$ & $43(72.9 \%)$ & 0.282 \\
\hline Current tobacco use & 39 (20.4\%) & $22(18.6 \%)$ & $17(23.3 \%)$ & 0.464 \\
\hline CCF & $34(17.8 \%)$ & $30(25.4 \%)$ & $4(5.5 \%)$ & $<0.001$ \\
\hline NYHA 3,4 & $20(10.5 \%)$ & $14(11.9 \%)$ & $6(8.2 \%)$ & 0.476 \\
\hline LVEF & $53 \pm 11$ & $51 \pm 10$ & $54 \pm 11$ & 0.076 \\
\hline Previous MI & $100(52.4 \%)$ & 65 (55.1\%) & 35 (47.9\%) & 0.373 \\
\hline Previous CABG & $14(7.3 \%)$ & $9(7.6 \%)$ & $5(6.8 \%)$ & 1.000 \\
\hline Statin therapy & $163(85.3 \%)$ & 99 (83.9\%) & $64(87.7 \%)$ & 0.533 \\
\hline \multicolumn{5}{|l|}{ Lipid profile* $(\mathrm{mg} / \mathrm{dL})$} \\
\hline Total cholesterol & $145 \pm 42$ & $146 \pm 45$ & $144 \pm 37$ & 0.785 \\
\hline LDL & $80 \pm 35$ & $80 \pm 38$ & $79 \pm 31$ & 0.896 \\
\hline $\mathrm{HDL}$ & $39 \pm 12$ & $41 \pm 13$ & $38 \pm 10$ & 0.126 \\
\hline Triglycerides & $130 \pm 71$ & $124 \pm 67$ & $140 \pm 76$ & 0.166 \\
\hline
\end{tabular}

ACS acute coronary syndrome, $B M I$ body mass index, CABG coronary artery bypass grafting, CCF congestive cardiac failure, LVEF left ventricular ejection fraction, LDL low-density lipoprotein, HDL high-density lipoprotein, $M I$ myocardial infarction, NYHA New York Heart Association

*Dyslipidemia defined as total cholesterol $>250 \mathrm{mg} / \mathrm{dL}$, LDL cholesterol $>130 \mathrm{mg} / \mathrm{dL}, \mathrm{HDL}$ cholesterol $<40 \mathrm{mg} / \mathrm{dL}$ ( $<50 \mathrm{mg} / \mathrm{dL}$ for women) in the fasting state. Data available for 146 patients

${ }^{t}$ Blood pressure $>140 / 90 \mathrm{mmHg}$ or the use of antihypertensive therapy

Angiographic and procedural characteristics of the initial PCI procedure can influence the development of ISR and potentially affect its clinical presentation. Such data was available in only 106 study patients as many of these patients presented for the first time to our hospital with ISR and/or medical records of initial PCI was unavailable. We have compared these variables among groups currently presenting with or without ACS (Table 4). These characteristics were not significantly associated with ACS ISR presentation.

\section{Effect of type of clinical presentation on outcomes}

Majority of the adverse events (24 out of 30 events, $80 \%$ ) during the 1-year follow-up period occurred in patients with ISR who presented with ACS during the index hospitalization. Kaplan-Meier survival curves for ACS and non-ACS presentations are shown in Fig. 2. ISR patients presenting with ACS had a 2.66-fold higher risk of MACE at 1 year (hazard ratio [HR], 2.66; 95\% CI, 1.09$6.50 ; P=0.032$ ). There was one case of definite stent thrombosis following $\mathrm{PCI}$ in the ACS group.
Within the ACS group, patients presenting with MI had a higher 1-year event rate compared to those who presented with unstable angina $(24.2 \%$ and $15.4 \%$ respectively), but the difference was not statistically significant.

In the overall cohort (191 patients), there was no difference in 1-year outcomes with respect to the treatment received (event rate in medical therapy vs. CABG vs. PCI, $16.2 \%$ vs. $14.0 \%$ vs. $16.3 \% ; P=0.928$ ).

\section{Discussion}

Our study is the first of its kind to explore DES ISR clinical presentation and outcomes irrespective of the treatment strategy used. The main findings of this study, which included 191 patients with 210 culprit ISR lesions, were (1) ACS is the most common clinical presentation of DES ISR, (2) female gender and chronic kidney disease correlated with ACS ISR presentation and (3) ACS presentation of ISR was associated with significantly higher mortality and MACE at 1-year follow-up compared to non-ACS presentation. 
Table 2 Angiographic characteristics and treatment characteristics at first clinical ISR presentation

\begin{tabular}{|c|c|c|c|c|}
\hline Parameter & Total & ACS cohort & Non-ACS cohort & $P$ value \\
\hline ISR characteristics & $(n=210)$ & $(n=129)$ & $(n=81)$ & \\
\hline ISR type & & & & 0.961 \\
\hline I. Focal & $134(63.8 \%)$ & $84(65.1 \%)$ & $50(61.7 \%)$ & \\
\hline II. Diffuse & $23(11.0 \%)$ & $14(10.9 \%)$ & $9(11.1 \%)$ & \\
\hline III. Proliferative & $10(4.7 \%)$ & $6(4.7 \%)$ & $4(4.9 \%)$ & \\
\hline IV. Complete & $43(20.5 \%)$ & $25(19.4 \%)$ & $18(22.2 \%)$ & \\
\hline ISR vessel & & & & 0.203 \\
\hline Left anterior descending & $112(53.3 \%)$ & $62(48.1 \%)$ & $50(61.7 \%)$ & \\
\hline Left circumflex artery & $49(23.3 \%)$ & $33(25.6 \%)$ & $16(19.8 \%)$ & \\
\hline Right coronary artery & $47(22.4 \%)$ & $32(24.8 \%)$ & $15(18.5 \%)$ & \\
\hline Left main & $2(1.0 \%)$ & $2(1.6 \%)$ & $0(0.0 \%)$ & \\
\hline Proximal ISR location & $113(53.8 \%)$ & $68(52.7 \%)$ & $45(55.6 \%)$ & 0.776 \\
\hline Disease burden & $(n=191)$ & $(n=118)$ & $(n=73)$ & 0.715 \\
\hline Single-vessel disease & $72(37.7 \%)$ & $42(35.6 \%)$ & $30(41.1 \%)$ & \\
\hline Double-vessel disease & $67(35.1 \%)$ & $42(35.6 \%)$ & $25(34.2 \%)$ & \\
\hline Triple-vessel disease & $52(27.2 \%)$ & $34(28.8 \%)$ & $18(24.7 \%)$ & \\
\hline Treatment plan & $(n=191)$ & $(n=118)$ & $(n=73)$ & 0.620 \\
\hline Medical therapy & 37 (19.4\%) & $24(20.3 \%)$ & $13(17.8 \%)$ & \\
\hline CABG & $50(26.2 \%)$ & $33(28.0 \%)$ & $17(23.3 \%)$ & \\
\hline $\mathrm{PCl}$ & $104(54.5 \%)$ & $61(51.7 \%)$ & $43(58.9 \%)$ & \\
\hline \multicolumn{5}{|l|}{ Details of $\mathrm{PCl}$} \\
\hline Procedural success & $101(97.1 \%)$ & $59(96.7 \%)$ & $42(97.7 \%)$ & 1.000 \\
\hline PCl type & & & & 0.082 \\
\hline POBA & $21(20.2 \%)$ & $16(26.2 \%)$ & $5(11.6 \%)$ & \\
\hline DCB & $11(10.6 \%)$ & $4(6.6 \%)$ & 7 (16.3\%) & \\
\hline New DES & $72(69.2 \%)$ & $41(67.2 \%)$ & $31(72.1 \%)$ & \\
\hline No. of stents & $1.14 \pm 0.35$ & $1.16 \pm 0.37$ & $1.12 \pm 0.33$ & 0.608 \\
\hline Stent length & $29.7 \pm 11.0$ & $32.0 \pm 12.9$ & $26.4 \pm 6.4$ & 0.045 \\
\hline Stent diameter & $3.03 \pm 0.40$ & $3.00 \pm 0.36$ & $3.08 \pm 0.45$ & 0.451 \\
\hline \multicolumn{5}{|l|}{ Adjunct Devices } \\
\hline Rotablation & $2(1.9 \%)$ & $2(3.3 \%)$ & $0(0.0 \%)$ & 0.342 \\
\hline Cutting or NC balloon & $24(23.1 \%)$ & $13(21.3 \%)$ & $11(25.6 \%)$ & 0.390 \\
\hline IVUS guidance & $25(24.0 \%)$ & $13(21.3 \%)$ & $12(27.9 \%)$ & 0.292 \\
\hline
\end{tabular}

ACS acute coronary syndrome, $C A B G$ coronary artery bypass grafting, $D C B$ drug-coated balloon, DES drug-eluting stent, IVUS intravascular ultrasound, ISR in-stent restenosis, $N C$ non-compliant, $P C I$ percutaneous coronary intervention, $P O B A$ plain old balloon angioplasty

\section{Clinical presentation of DES-ISR}

Our study found that ACS (62\% of patients) is the dominant presentation mode for DES-ISR and 34\% of patients presented with MI. An acute unstable presentation was seen in up to $70 \%$ of patients with ISR in both BMS and DES eras of whom $10-20 \%$ presented with MI [11, 12, 19-23]. A recent study showed that ACS is the common ISR presentation mode across three stent generations but suggested that second-generation DES may present less often with MI [11]. In addition to this, the risk of late stent thrombosis with DES, albeit small, adds to this problem [24]. Despite the advantages of DES over BMS in reducing the incidence of ISR, the propensity of ISR to present with ACS has remained largely similar irrespective of stent type and has important therapeutic and prognostic implications.

\section{Mechanism of ACS ISR presentation}

An enhanced local inflammatory reaction, fibroatheromas with thin caps, higher lipid content in plaques and/or superimposed thrombus may contribute to ACS ISR presentation with DES. Several studies have confirmed these theories by demonstrating thrombi overlying neointimal 
Table 3 Correlates of ACS ISR presentation compared to non-ACS presentation

\begin{tabular}{|c|c|c|c|c|c|c|c|c|}
\hline \multirow[t]{2}{*}{ Variables } & \multicolumn{4}{|c|}{ Univariate analysis } & \multicolumn{4}{|c|}{ Multivariate analysis } \\
\hline & OR & Lower 95\% Cl & Upper 95\% Cl & $P$ value & OR & Lower 95\% Cl & Upper 95\% Cl & $P$ value \\
\hline \multicolumn{9}{|l|}{ Patient-related } \\
\hline Age & 1.03 & 0.99 & 1.06 & 0.076 & 1.01 & 0.98 & 1.05 & 0.449 \\
\hline Female gender & 2.53 & 1.08 & 5.90 & 0.032 & 2.71 & 1.13 & 6.52 & 0.026 \\
\hline BMI & 1.04 & 0.93 & 1.17 & 0.476 & - & - & - & - \\
\hline Diabetes & 1.06 & 0.59 & 1.91 & 0.843 & - & - & - & - \\
\hline Hypertension & 1.17 & 0.65 & 2.10 & 0.601 & - & - & - & - \\
\hline Current tobacco use & 0.76 & 0.37 & 1.54 & 0.440 & - & - & - & - \\
\hline Dyslipidemia & 1.56 & 0.76 & 3.21 & 0.224 & - & - & - & - \\
\hline Chronic kidney disease & 4.20 & 1.19 & 14.80 & 0.026 & 3.85 & 1.05 & 14.20 & 0.043 \\
\hline CCF & 5.88 & 1.98 & 17.49 & 0.001 & 4.98 & 1.63 & 15.26 & 0.005 \\
\hline Previous Ml & 1.33 & 0.74 & 2.39 & 0.338 & - & - & - & - \\
\hline Previous CABG & 1.12 & 0.36 & 3.49 & 0.841 & - & - & - & - \\
\hline Statin therapy & 0.73 & 0.31 & 1.72 & 0.475 & & & & \\
\hline \multicolumn{9}{|l|}{ Lesion-related } \\
\hline LAD involvement & 0.71 & 0.39 & 1.28 & 0.248 & - & - & - & - \\
\hline Proximal ISR location & 0.88 & 0.49 & 1.58 & 0.656 & - & - & - & - \\
\hline
\end{tabular}

$A C S$ acute coronary syndrome, $B M I$ body mass index, CABG coronary artery bypass grafting, CCF congestive cardiac failure, $C I$ confidence interval, ISR in-stent restenosis, $L A D$ left anterior descending, $L V E F$ left ventricular ejection fraction, $M I$ myocardial infarction, $O R$ odds ratio

Table 4 Angiographic and PCl characteristics during initial PCl

\begin{tabular}{|c|c|c|c|c|}
\hline Parameter & Total $(n=106)$ & ACS cohort $(n=68)$ & Non-ACS cohort $(n=38)$ & $P$ value \\
\hline Lesion type & & & & 0.427 \\
\hline A & $51(48.1 \%)$ & $29(42.7 \%)$ & 22 (57.9\%) & \\
\hline B & $28(26.4 \%)$ & 19 (27.9\%) & $9(23.7 \%)$ & \\
\hline C & $27(25.5 \%)$ & $20(29.4 \%)$ & $7(18.4 \%)$ & \\
\hline Calcification & $14(13.2 \%)$ & $7(10.3 \%)$ & $7(18.4 \%)$ & 0.358 \\
\hline Disease burden & & & & 0.627 \\
\hline Single-vessel disease & 55 (51.9\%) & 38 (55.9\%) & $17(44.7 \%)$ & \\
\hline Double-vessel disease & $33(31.1 \%)$ & $18(26.5 \%)$ & 15 (39.5\%) & \\
\hline Triple-vessel disease & $18(17.0 \%)$ & $12(17.6 \%)$ & $6(15.8 \%)$ & \\
\hline \multicolumn{5}{|l|}{ Details of $\mathrm{PCl}$} \\
\hline No. of stents & $1.3 \pm 0.6$ & $1.3 \pm 0.6$ & $1.3 \pm 0.6$ & 0.923 \\
\hline Stent length & $27.0 \pm 8.7$ & $28.2 \pm 8.7$ & $24.6 \pm 8.6$ & 0.124 \\
\hline Stent diameter & $2.92 \pm 0.34$ & $2.93 \pm 0.36$ & $2.90 \pm 0.31$ & 0.712 \\
\hline Pre-dilation & $63(59.4 \%)$ & $43(63.2 \%)$ & $20(52.6 \%)$ & 0.541 \\
\hline Post-dilation & $43(40.5 \%)$ & $27(39.7 \%)$ & $16(42.1 \%)$ & 0.944 \\
\hline DES type & & & & 0.683 \\
\hline Paclitaxel & $3(2.8 \%)$ & $3(4.4 \%)$ & - & \\
\hline Sirolimus & $70(66.1 \%)$ & $46(67.6 \%)$ & $24(63.2 \%)$ & \\
\hline Everolimus & $21(19.8 \%)$ & $12(17.7 \%)$ & $9(23.7 \%)$ & \\
\hline Zotarolimus & $12(11.3 \%)$ & $7(10.3 \%)$ & $5(13.1 \%)$ & \\
\hline Time to ISR & $26.8 \pm 25$ & $24.5 \pm 24$ & $31.9 \pm 30.1$ & 0.481 \\
\hline
\end{tabular}




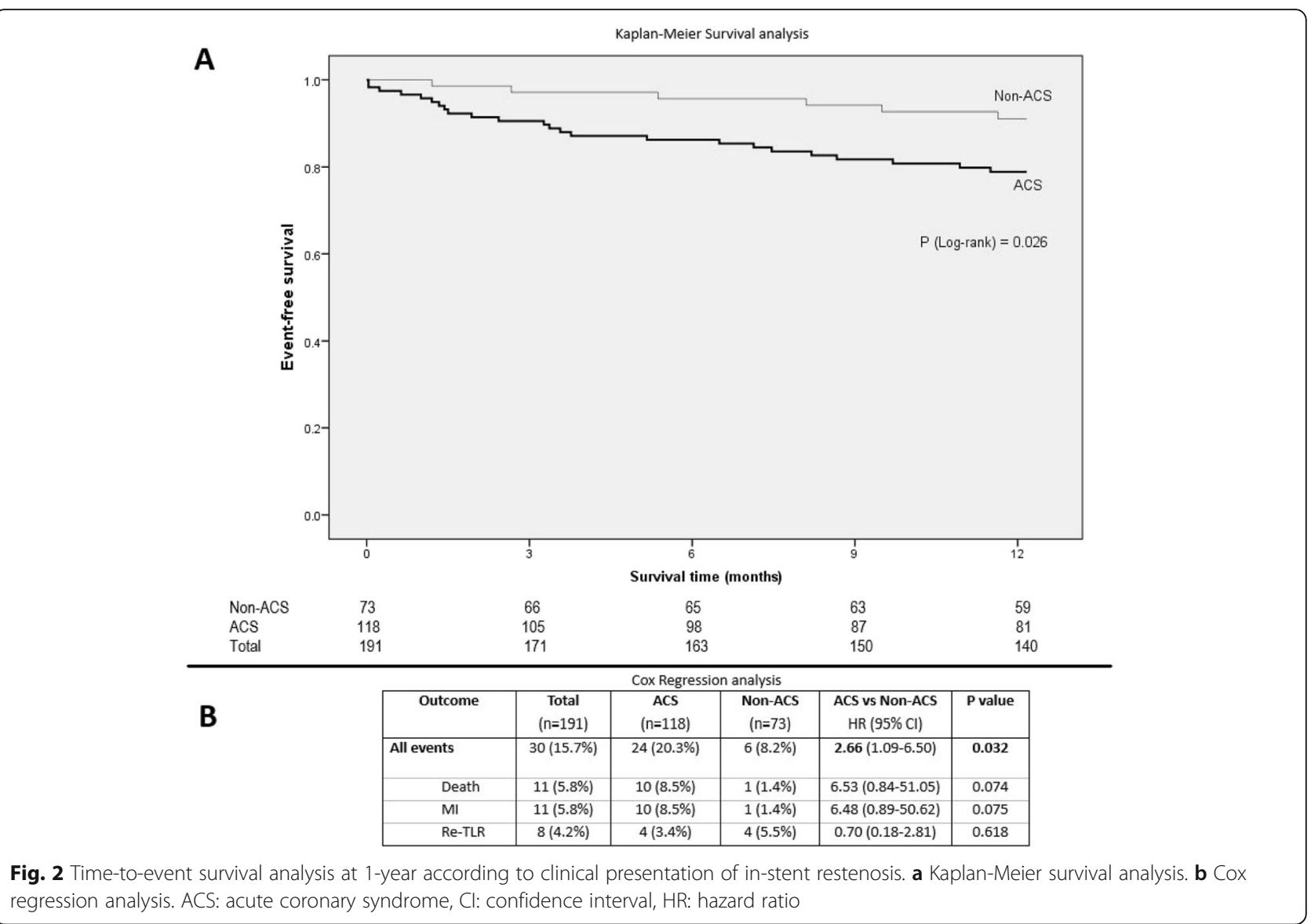

disruptions using intravascular imaging or by demonstrating fibrin/thrombi in ISR tissue of patients presenting with ACS $[25,26]$. Some studies have suggested that, compared to first-generation DES, second-generation DES are associated with better vascular healing, lower prevalence of neoatherosclerosis and reduction in the incidence of unstable features such as disrupted neointima, thin-cap fibroatheroma, thrombus and fibrin deposition [27, 28]. In our setting, a plethora of stent types with various combinations of anti-proliferative drugs and polymers are available which makes it difficult to analyse the results stratified by stent types [29]. Mechanisms underlying ACS presentations with various stent types need further studies.

\section{Clinical correlates of DES-ISR presentation}

Our study found that female gender and chronic kidney disease are significantly associated with ACS presentation. Further, patients presenting with ACS are more likely to have congestive heart failure compared to those presenting with stable syndromes. However, we did not find an association between age, body mass index, current tobacco use, history of previous MI or CABG, diabetes, hypertension or dyslipidemia and ACS presentation. Type of ISR and its location also did not correlate with presentation mode.

Women are known to present more often with atypical chest pain and angina equivalents such as dyspnea, fatigue, indigestion and weakness which may lead to delayed diagnosis and management of coronary disease [30-32]. However, these studies were conducted in women at first presentation of ischemic heart disease (IHD). Patients who present with ISR are already under treatment for IHD which makes it less likely that atypical symptoms would be ignored. This is probably the reason why none of the previous studies has found the patient's gender to be related to the ISR presentation mode [11, 12]. Reasons for women with ISR presenting more often as ACS in our study are unclear. Women's health receives less attention compared to their male counterparts especially in developing countries and therefore may not receive medical attention unless a more dramatic presentation ensues [33-36]. Whether such sociocultural factors contributed to our study finding needs further exploration.

Chronic kidney disease has been shown to be associated with poor outcomes after PCI with both BMS and DES era. It was found to be a factor independently 
associated with ISR presenting as MI [11]. This is likely explained by the increased incidence of neoatherosclerosis and higher lipid content in neointima among patients with CKD [28, 37].

Some studies found smoking to be associated with ACS ISR presentation [11]. Our study did not find an association between tobacco use and ACS presentation even after adjustment for gender differences. Differences in the way tobacco is consumed by the study population (smoking vs. chewing) and a possible reduction in the quantity of tobacco consumed due to repeated counselling during clinical visits may underlie these findings.

Although the development of ISR per se has been attributed to patient-related, stent-related and technical factors, clinical presentation mode appears to be related to patient-related factors alone $[11,12]$. It is therefore important to identify patients who are at higher risk of ACS ISR presentation. Women who receive DES, and their caregivers, need to be counselled regarding regular clinical follow-up and the importance of seeking timely medical attention.

\section{Clinical outcomes}

In our study, the clinical presentation mode affected patient outcomes. ACS ISR presentation was independently associated with a higher incidence of composite clinical outcome of death, MI and re-TLR at 1-year follow-up compared to a non-ACS presentation. Similar findings were reported by several previous studies on both BMS and DES restenosis [11, 12, 21, 22, 38, 39]. At least one previous study even reported that ISR presentation as MI may be worse than stent thrombosis [40].

ACS presentation was shown to be associated with a higher incidence of MACE and TLR in BMS ISR in the PRESTO trial [21]. Similarly, a higher risk of re-TLR was seen when patients with a first-generation DES presented with unstable angina compared to stable syndromes [38]. One study even suggested that DES ISR may be associated with poorer outcomes compared to BMS ISR for an identical level of cardiac risk [12]. Recently, a large study concluded that ACS ISR presentation is a harbinger of worse outcomes across all three stent generations (BMS, first- and second-generation DES) [11]. It is therefore pertinent to identify those at risk of ACS presentation and to closely follow ISR patients who presented with an ACS. Novel treatment strategies may be needed to improve the outcomes of patients with ACS ISR presentation.

In our entire DES-ISR cohort (191 patients), no difference in outcomes at 1-year follow-up was seen between groups receiving medical therapy, CABG and PCI. The treatment strategy was solely based on the physician's discretion which is an important confounding factor. Therefore, this finding cannot be used to conclude that all treatment strategies are equally effective in DES ISR. We could not come across any study comparing medical therapy, CABG and PCI for DES ISR. Further research using a randomized controlled trial design are needed to compare outcomes among different treatment modalities.

\section{Limitations}

This is a retrospective observational study, and therefore, the results may be affected by various confounding factors. The findings of this study should, therefore, be considered hypothesis-generating.

Despite the rigorous process of adjudication used, the possibility of late stent thrombosis masquerading as ISR with MI cannot be excluded. Recent studies with intravascular imaging have suggested that ISR and stent thrombosis may not be entirely distinct clinical entities.

Type of DES (first- vs. second-generation DES) received by study patients in their initial procedure (prior to the development of ISR) could not be ascertained in all patients. Therefore, the impact of the type of DES on clinical presentation could not be compared. However, in developing countries like ours, a variety of stent types with various combinations of anti-proliferative drugs and polymers are available which makes it difficult to segregate them into two or three groups for study purposes [29].

Treatment modalities could not be compared because patients were treated according to physician discretion with either PCI, CABG or medical management. Because re-TLR cannot occur in the latter two groups, re-TLR rates in our study are consequently lower. Further, the type of PCI (new DES, DCB or POBA) may also have influenced outcomes. However, we believe our study is representative of the entire spectrum of clinical ISR in the real-world situation where numerous factors affect treatment decisions and outcomes.

\section{Conclusions}

DES-ISR presents more often as ACS, and patientrelated factors like female gender and chronic kidney disease are associated with an ACS presentation. ACS presentation is independently associated with poorer clinical outcomes.

In-stent restenosis continues to be an important problem even in the current DES era because of the propensity to present as ACS. Close monitoring of patients with ACS ISR presentations is necessary. Finding and addressing the causes of gender differences in the clinical presentation of ISR may improve clinical outcomes among women. There is a need for new approaches or technologies to completely eradicate the problem of ISR.

\section{Abbreviations}

ACS: Acute coronary syndrome; ASCVD: Atherosclerotic cardiovascular disease; BMS: Bare-metal stent; CABG: Coronary-artery bypass grafting; 
CCF: Congestive cardiac failure; DES: Drug-eluting stent; ISR: In-stent restenosis; MACE: Major adverse cardiac events; MI: Myocardial infarction; PCI: Percutaneous coronary intervention; TLR: Target lesion revascularization

\section{Acknowledgements}

The authors acknowledge Dr. Indu Ramachandra Rao for proof-reading the article and her valuable inputs.

\section{Authors' contributions}

Research idea, study design and statistical analysis were done by GP and TD. Data collection was done predominantly by GP, SU, AS, UP, KN and MSR. Data analysis and interpretation was carried out by all authors. The article was written predominantly by GP. All authors read and approved the final manuscript.

\section{Funding}

This research did not receive any specific grant from funding agencies in the public, commercial or not-for-profit sectors.

\section{Availability of data and materials}

The dataset supporting the results and conclusions of this article will be available from the corresponding author on request.

\section{Ethics approval and consent to participate}

The study protocol was approved by the Kasturba Medial College and Kasturba Hospital Institutional Ethics Committee (IEC number: 351/2018). Consent to participate is not applicable as this is a retrospective study.

\section{Consent for publication}

Not applicable.

\section{Competing interests}

The authors declare that they have no competing interests.

\section{Author details}

'Department of Cardiology, Kasturba Medical College, Manipal, Manipa Academy of Higher Education, Manipal, Karnataka 576104, India. 2Department of Cardiovascular Technology, School of Allied Health Sciences, Manipal Academy of Higher Education, Manipal, Karnataka 576104, India.

\section{Received: 22 August 2019 Accepted: 1 October 2019}

\section{Published online: 27 November 2019}

\section{References}

1. Camenzind $E$ (2006) Treatment of in-stent restenosis - back to the future? N Engl J Med 355:2149-2151

2. Serruys PW, de Jaegere P, Kiemeneij F et al (1994) A comparison of balloonexpandable-stent implantation with balloon angioplasty in patients with coronary artery disease. N Engl J Med 331:489-495

3. Hoffmann R, Mintz GS, Dussaillant GR et al (1996) Patterns and mechanisms of in-stent restenosis. Circulation 94:1247-1254

4. Virmani R, Farb A (1999) Pathology of in-stent restenosis. Curr Opin Lipidol 10:499-506

5. Morice M-C, Serruys PW, Sousa JE et al (2002) A randomized comparison of a sirolimus-eluting stent with a standard stent for coronary revascularization. N Engl J Med 346:1773-1780

6. Stone GW, Ellis SG, Cox DA et al (2004) A polymer-based, paclitaxel-eluting stent in patients with coronary artery disease. N Engl J Med 350:221-23

7. Stettler C, Wandel S, Allemann S et al (2007) Outcomes associated with drug-eluting and bare-metal stents: a collaborative network meta-analysis. Lancet 370:937-948

8. Cassese S, Byrne RA, Tada T et al (2014) Incidence and predictors of restenosis after coronary stenting in 10004 patients with surveillance angiography. Heart 100:153-159

9. Mauri L, Silbaugh TS, Wolf RE et al (2008) Long-term clinical outcomes after drug-eluting and bare-metal stenting in Massachusetts. Circulation 118 : 1817-1827

10. Corbett SJ, Cosgrave J, Melzi G et al (2006) Patterns of restenosis after drugeluting stent implantation: insights from a contemporary and comparative analysis of sirolimus- and paclitaxel-eluting stents. Eur Heart J 27:2330-2337
11. Magalhaes MA, Minha S, Chen F et al (2014) Clinical presentation and outcomes of coronary in-stent restenosis across 3-stent generations. Circ Cardiovasc Interv 7:768-776

12. De Labriolle A, Bonello L, Lemesle G et al (2009) Clinical presentation and outcome of patients hospitalized for symptomatic in-stent restenosis treated by percutaneous coronary intervention: comparison between drugeluting stents and bare-metal stents. Arch Cardiovasc Dis 102:209-217

13. Mehran R, Dangas G, Abizaid AS et al (1999) Angiographic patterns of instent restenosis: classification and implications for long-term outcome. Circulation 100:1872-1878

14. Alfonso F, Zueco J, Cequier A et al (2003) A randomized comparison of repeat stenting with balloon angioplasty in patients with in-stent restenosis. J Am Coll Cardiol 42:796-805

15. Thygesen K, Alpert JS, Jaffe AS et al (2018) Fourth universal definition of myocardial infarction (2018). Circulation 138:e618-e651

16. Montalescot G, Sechtem U, Achenbach S et al (2013) 2013 ESC guidelines on the management of stable coronary artery disease: the task force on the management of stable coronary artery disease of the European Society of Cardiology. Eur Heart J 34:2949-3003

17. Levin A, Stevens PE, Bilous RW et al (2013) Kidney disease: improving global outcomes (KDIGO) CKD work group. KDIGO 2012 clinical practice guideline for the evaluation and management of chronic kidney disease. Kidney Int Suppl 3:1-150. https://doi.org/10.1038/kisup.2012.73

18. Cutlip DE, Windecker S, Mehran R et al (2007) Clinical end points in coronary stent trials: a case for standardized definitions. Circulation 115: 2344-2351

19. Chen MS, John JM, Chew DP et al (2006) Bare metal stent restenosis is not a benign clinical entity. Am Heart J 151:1260-1264

20. Nayak AK, Kawamura A, Nesto RW et al (2006) Myocardial infarction as a presentation of clinical in-stent restenosis. Circ J 70:1026-1029

21. Assali AR, Moustapha A, Sdringola S et al (2006) Acute coronary syndrome may occur with in-stent restenosis and is associated with adverse outcomes (the PRESTO trial). Am J Cardiol 98:729-733

22. Doyle B, Rihal CS, O'Sullivan CJ et al (2007) Outcomes of stent thrombosis and restenosis during extended follow-up of patients treated with baremetal coronary stents. Circulation 116:2391-2398

23. Schwalm T, Carlsson J, Meissner A et al (2013) Current treatment and outcome of coronary in-stent restenosis in Sweden: a report from the Swedish coronary angiography and angioplasty registry (SCAAR). Eurolntervention 9:564-572

24. Daemen J, Wenaweser P, Tsuchida K et al (2007) Early and late coronary stent thrombosis of sirolimus-eluting and paclitaxel-eluting stents in routine clinical practice: data from a large two-institutional cohort study. Lancet 369:667-678

25. Kang S-J, Mintz GS, Akasaka T et al (2011) Optical coherence tomographic analysis of in-stent neoatherosclerosis after drug-eluting stent implantation. Circulation 123:2954-2963

26. Oikawa Y, Yajima J, Costa M et al (2010) Intravascular ultrasound, angioscopic and histopathological characterisation of heterogeneous patterns of restenosis after sirolimus-eluting stent implantation: insights into potential "thromborestenosis" phenomenon. Eurolntervention 6:380-387

27. Otsuka F, Vorpahl M, Nakano M et al (2014) Pathology of second-generation everolimus-eluting stents versus first-generation Sirolimus- and paclitaxeleluting stents in humans. Circulation 129:211-223

28. Yonetsu T, Kato K, Kim S-J et al (2012) Predictors for neoatherosclerosis. Circ Cardiovasc Imaging 5:660-666

29. Sastry BKS, Nallamalla KR, Kumar N et al (2018) One-year clinical outcomes of different coronary drug eluting stents_-data from a prospective registry. Indian Heart J 70:580-583

30. Wenger NK (2010) Angina in women. Curr Cardiol Rep 12:307-314

31. Khan NA, Daskalopoulou SS, Karp I et al (2013) Sex differences in acute coronary syndrome symptom presentation in young patients. JAMA Intern Med 173:1863-1871

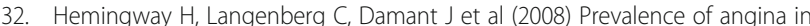
women versus men: a systematic review and meta-analysis of international variations across 31 countries. Circulation 117:1526-1536

33. Kaur R, Lopez V, Thompson DR (2006) Factors influencing Hong Kong Chinese patients' decision-making in seeking early treatment for acute myocardial infarction. Res Nurs Health 29:636-646

34. DeVon HA (2011) Promoting cardiovascular health in women across the life span. J Obstet Gynecol Neonatal Nurs 40:335-336 
35. Lichtman JH, Leifheit-Limson EC, Watanabe E et al (2015) Symptom recognition and healthcare experiences of young women with acute myocardial infarction. Circ Cardiovasc Qual Outcomes 8:S31-S38

36. Pagidipati NJ, Peterson ED (2016) Acute coronary syndromes in women and men. Nat Rev Cardiol 13:471-480

37. Hayano S, Ishii H, Ichimiya S et al (2013) Renal dysfunction and atherosclerosis of the neointima following bare metal stent implantation. Am J Nephrol 38:58-65

38. Rathore S, Kinoshita Y, Terashima M et al (2010) A comparison of clinical presentations, angiographic patterns and outcomes of in-stent restenosis between bare metal stents and drug eluting stents. Eurolntervention 5:841-846

39. lijima R, Araki T, Nagashima Y et al (2013) Incidence and predictors of the late catch-up phenomenon after drug-eluting stent implantation. Int J Cardiol 168:2588-2592

40. Hayes KR, Applegate RJ, Sacrinty MT et al (2010) Target lesion revascularization after bare-metal or drug-eluting stents: clinical presentations and outcomes. J Invasive Cardiol 22:266-270

\section{Publisher's Note}

Springer Nature remains neutral with regard to jurisdictional claims in published maps and institutional affiliations.

\section{Submit your manuscript to a SpringerOpen ${ }^{\circ}$ journal and benefit from:}

- Convenient online submission

- Rigorous peer review

- Open access: articles freely available online

- High visibility within the field

- Retaining the copyright to your article 